\title{
Novel Options and Limitations of Methanol Based Production and Storage for Mobile Applications
}

\author{
D. Poetzsch, ${ }^{1 \&}$ T. Bach, ${ }^{1 \S}$ J.E. Zerpa Unda, ${ }^{1 \uparrow}$ E. Roduner, ${ }^{1,2 *}$ \\ ${ }^{1}$ Institute of Physical Chemistry, University of Stuttgart, Pfaffenwaldring 55, D-70569 Stuttgart, Germany \\ ${ }^{2}$ Chemistry Department, University of Pretoria, Pretoria 0002, Republic of South Africa
}

Received

[*] Corresponding author, e.roduner@ipc.uni-stuttgart.de

[\$] Present address: Fraunhofer Institute for Silicate Research, Neunerplatz 2, D-97082 Würzburg, Germany

[\&] Present address: Max-Planck-Institute for Solid State Research, Heisenbergstraße 1, D-70569 Stuttgart, Germany

[ף] Present address: BASF SE, GCN/EP - M311, D-67056 Ludwigshafen, Germany

\begin{abstract}
Methanol is considered a promising liquid fuel in context with electrochemical energy conversion and storage for mobile applications. It is shown here that a direct methanol fuel cell can be used for spontaneous charging and discharging a supercapacitor for intermediate storage of chemical energy. Thereby, protons and electrons of the methanol-derived hydrogen are stored separately in the electrical double layer of the supercapacitor electrode. The charging and discharging of this fuel cell-supercapacitor hybride device is investigated in experiments of spontaneous conditions (closed circuit) and also under externally enforced constant voltage sweep rate (cyclic voltammetry) and under constant current conditions. Alternatively, gas phase hydrogen is generated from methanol in an electroreforming process. When more efficient anode electrocatalysts become available this may become the method of choice for on-board and on demand hydrogen production in mobile applications.
\end{abstract}

Keywords: Electrochemical Hydrogen Storage, DMFC-Supercapacitor Hybrid Device, Methanol Electro-Reforming, Charging of Porous Electrode

\section{Introduction}

The prospects of the consequences of the increasing world energy demand, the continuing rise of the carbon dioxide and other greenhouse gas content in the earth atmosphere and the impact of major accidents with fossil fuel handling and nuclear energy production plants have led to tremendous support in the opinion that there is a need for alternative energy resources and new general energy technology. A significant problem arises because of the strongly cyclic demand of energy on various timescales, depending on the application; at the same time many of the alternative energy resources depend on day-night or annual periodicity or on weather and are thus also cyclic, but uncorrelated with demand. Therefore, energy storage on timescales of milliseconds to months is just as important an issue as energy conversion. Large scale storage is best done as chemical energy.

The successful strategy under conditions of cyclic demand is often based on hybrid operation of more than one power source. The base power can be covered by a sluggish source, while peak power is provided flexibly by a fast converter or a storage medium which is recharged in times of low need. Fuel cells, for example, are best operated in a relatively narrow current-voltage range in order to minimize degradation processes which affect membrane and electrodes. They can be complemented with batteries which in the past decade have made significant progress regarding energy density. If a fast response is needed a supercapacitor may be a better solution, but with limited energy density. The complementary devices are often wired up in parallel. Here we show that a more integrated design is basically also possible.

Hydrogen is the chemical energy carrier with the highest gravimetric energy density, but since it is a gas, storage and transport are inconvenient for mobile applications. Various ways of hydrogen storage are being investigated, among them liquefaction at $20 \mathrm{~K}$, compression in pressure tanks at up to 700 bar, physisorption on large surface area carbon or molecular framework materials (MOFs), chemical storage in metal hydrides or in alanates, aminoborane, or their amidoborane derivatives, or borohydrides [1,2]. Recently, $\mathrm{H}_{2}$ physisorption on lithium dodecahydro-dodeca-closoborane, $\mathrm{Li}_{2} \mathrm{~B}_{12} \mathrm{H}_{12}$ was suggested as a novel option for hydrogen storage [3]. It was shown that the entropy associated with volume compression to an adsorbed, condensed or simply compacted state requires energy corresponding to on the order of $15 \%$ of the hydrogen lower heating value. This entropy term thus represents a fundamental barrier hindering reversible storage.

Recent work has explored a novel concept of hydrogen storage in which protons and electrons are electrochemically separated on a polymer electrolyte membrane fuel cell-type electrode and then stored in the electrical double layer (EDL) of a supercapacitor-type electrode [4]. This is a spontaneous process at 1 bar $\mathrm{H}_{2}$ pressure and ambient 
temperature. The hydrogen was recovered and directly converted to water at the same electrode, after the hydrogen atmosphere had been replaced by oxygen. This is also a spontaneous and energy producing process. In essence, the device is a hybrid of a hydrogen fuel cell with an integrated hydrogen buffer. The present work extends this concept to using methanol as a fuel in place of hydrogen, and it has two separate fuel cell-type electrodes which permit simultaneous or sequential operation of the anode and cathode, depending on demand. The EDL supercapacitor is located between and wired up in series with anode and cathode. As a potential practical application one might envisage the operation of a laptop which for much of its time has a low energy demand that is well covered by the sluggish anode reaction of methanol, allowing at the same time the buffer to be filled. At times of peak demand, for example during writing of a $\mathrm{CD}$, the additional power is retrieved from the buffer.

Instead of storing hydrogen, it may be prepared on-board from methanol or gasoline by a thermal steam reforming process. This requires a chemical reactor at a temperature of $250-360^{\circ}$ and a pressure of ca. 20 bar, which poses special start-up procedures. Furthermore, the formation of $\mathrm{CO}$ has to be kept below ca. $50 \mathrm{ppm}$. These complications have prompted some research organizations to disregard thermal reforming for automotive power trains [5]. Nevertheless, several Reformed Methanol Fuel cells (RMFC) designs have reached commercial status in niche applications. Here we evaluate further a recently reported alternative [6] that has not received much attention in literature so far. It consists in the electrochemical conversion of aqueous methanol to $\mathrm{H}_{2}$ and $\mathrm{CO}_{2}$, which is the reaction that in principle occurs when the oxygen at the cathode of a direct methanol fuel cell is replaced by an inert gas so that the reaction stops at the stage of $\mathrm{H}_{2}$ without reacting further to water. With $\Delta G^{\circ}=-15 \mathrm{~kJ} \mathrm{~mol}^{-1}$ the reaction is slightly exergonic and would occur spontaneously if it were not highly activated. A suitable catalyst can reduce the activation energy of a homogeneous reaction, but if conducted in a fuel cell type setup the reaction can be promoted further by application of an external voltage. It is therefore called electro-reforming. As for thermal steam reforming, methanol is carried along as a liquid fuel in mobile applications. The process of electro-reforming, however, should allow for much simpler construction designs and operation.

\section{Carbon Based Supercapacitor, Spontaneously Charged and Discharged by a DMFC}

\subsection{The Concept}

With the exception of the metal hydrides, hydrogen is stored as a molecular entity, or as $\mathrm{H}$ atoms which are engaged in covalent chemical bonds. These reactions are far from being reversible. However, the standard hydrogen electrode tells us that gas phase hydrogen can be in equilibrium with solvated protons and compensating anions in water. Equilibration is fast and reversible at ambient temperature and pressure.

We have therefore explored the possibility to separate protons and electrons on a fuel cell type anode and store the two particles separately on an electrical double layer (EDL) supercapacitor, i.e. the electrons on large surface area carbon in contact with water where the solvated protons form the second half of the electrical double layer [4]. The discharge occurs at the cathode side of the fuel cell. The previous work used $\mathrm{H}_{2}$ as a fuel [4], but here we explore using a direct methanol fuel cell (DMFC) with an EDL supercapacitor integrated in a split membrane.

A schematic set-up is shown in Figure 1 which distinguishes three electrodes: a DMFC anode and cathode and a supercapacitor electrode. This system operates as a conventional DMFC when the supercapacitor electrode is disconnected, i.e. when the switches of the upper left and right circuits of the Figure are open. Under these conditions a specific constant current $\left(I_{\mathrm{FC}}\right)$ that originates from the fuel cell flows through the lower circuit. It depends on the performance of the cell and the internal resistance.

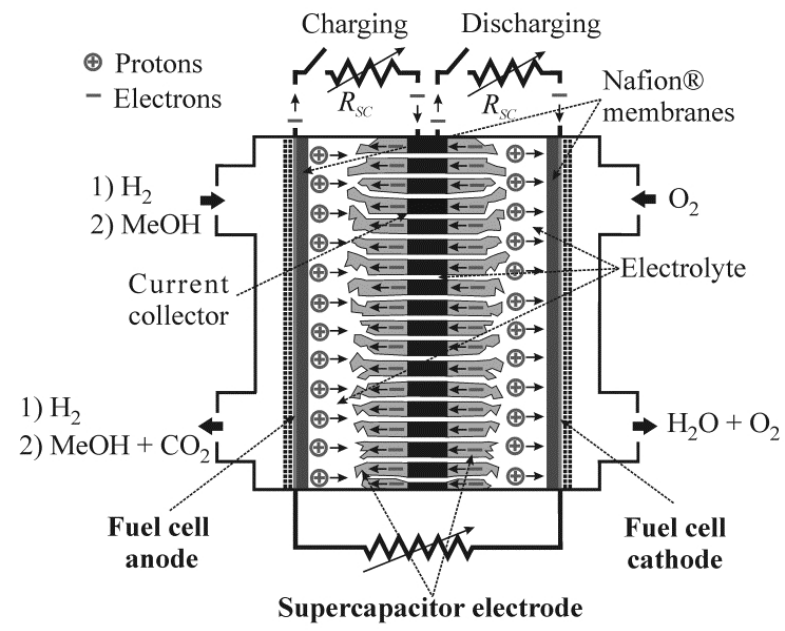

Figure 1: Scheme of hydrogen or methanol fuel cell with electrically contacted EDL supercapacitor integrated into the membrane (center of Figure). The left side of this hybrid device represents the charging at the anode, the right side the discharging of the capacitor at the fuel cell cathode. 
Specifically, protons and electrons are produced at the anode from the electrochemical oxidation of methanol during the charging process. The protons migrate from the anode through a Nafion ${ }^{\circledR}$ membrane towards the aqueous side of the supercapacitor electrode. At the same time the electrons flow through left half of the external circuit, producing electrical work. In the charged state protons are stored in the aqueous electrolyte side and electrons in the carbon material, thereby forming the double layer. During the discharging process the protons diffuse from the supercapacitor electrode through a second Nafion ${ }^{\circledR}$ membrane towards the cathode, where they react with oxygen and electrons to produce water and heat. Electrical work can be obtained during both processes.

It is important to highlight that the charging and discharging of this system is performed without the application of any external electrical current or voltage. The system is much like a battery where an open circuit voltage (OCV) can be measured between the anode and the supercapacitor electrode just before charging and between the cathode and the latter after charging (just before discharging). The origin of this OCV, which is the driving force for the chargingdischarging process, is related to thermodynamic reasons and also to the presence of electroactive functionalities at the carbon surface such as phenol, carbonyl, carboxyl, lactone, and the redox couple quinone/hydroquinone (Q/HQ) [7]. These surface functionalities are associated to the pseudocapacitive behavior exhibited by carbon electrodes [8]. As a consequence the total capacitive current measured during the charging-discharging process has two main contributions: a non-Faradaic, which involves the charging of the double layer, and a Faradaic associated to the potential dependent pseudocapacitive behavior of the carbon electrode.

Basically two charging-discharging processes are considered:

- One related to the capacitance:

$$
\begin{array}{ll}
\mathrm{MeOH}+7 \mathrm{H}_{2} \mathrm{O}+\{\mathrm{C}\} \rightleftharpoons 6 \mathrm{H}_{3} \mathrm{O}^{+}+\{\mathrm{C}\}^{-6}+\mathrm{CO}_{2} & \text { (Charging) } \\
6 \mathrm{H}_{3} \mathrm{O}^{+}+\{\mathrm{C}\}^{-6}+3 / 2 \mathrm{O}_{2} \rightleftharpoons\{\mathrm{C}\}+9 \mathrm{H}_{2} \mathrm{O} & \text { (Discharging) } \\
\text { and a reaction related to the pseudocapacitance: } & \text { (Charging) } \\
\mathrm{MeOH}+\mathrm{H}_{2} \mathrm{O}+6>\mathrm{C}=\mathrm{O} \rightleftharpoons 6>\mathrm{C}-\mathrm{OH}+\mathrm{CO}_{2} & \text { (Discharging) } \\
6>\mathrm{C}-\mathrm{OH}+3 / 2 \mathrm{O}_{2} \rightleftharpoons 6>\mathrm{C}=\mathrm{O}+3 \mathrm{H}_{2} \mathrm{O} &
\end{array}
$$

Here, the redox reactions of pseudocapacitive functional groups are represented by $>\mathrm{C}=\mathrm{O}$ and $>\mathrm{C}-\mathrm{O}$ which in this case is associated to the $\mathrm{Q} / \mathrm{HQ}$ pair. The symbol $\{\mathrm{C}\}$ refers to the carbon black electrode surface at which the double layer is formed.

\subsection{Experimental Details}

\subsubsection{Preparation of the Cell}

For all measurements we used a homemade, acrylic cell. The cathode side was actively supplied by pure oxygen; the anode side passively by an aqueous methanol solution in a tank. Therefore, we call this DMFC semi-passive.

On both sides of the supercapacitor (SC) part a membrane electrode assembly (MEA) with the electrode on only one side of the membrane was fixed. For the MEAs a gas diffusion layer (hydrophobic carbon paper from Freudenberg) was painted with a catalyst ink in thin layers at $80{ }^{\circ} \mathrm{C}$. The ink for the cathode contains carbon supported Pt (Alfa Aesar, 20 wt.\%), water and Nafion ${ }^{\circledR}$ ionomer suspension (Sigma-Aldrich, $10 \mathrm{wt} . \%$ ). The anode ink used supported Pt/Ru (Alfa Aesar, 40 wt.\% Pt, 20 wt.\% Ru) instead and additionally ethylene glycol for better adhesion [9]. The final metal loadings were $4.3 \mathrm{mg} \mathrm{cm}^{-2}$ and $1.8 \mathrm{mg} \mathrm{cm}^{-2}$ for the anode and cathode, respectively. These gas diffusion electrodes (GDE) were directly mounted together with Nafion ${ }^{\circledR} 117$ membranes in the cell without hot pressing [10-13].

The SC consists of a graphite substrate $(20 \times 20 \mathrm{~mm})$ with 100 holes $(1 \mathrm{~mm}$ diameter $)$ as current collector. This plate was coated with a paste of carbon black (Black Pearls 2000, CABOT Corporation) and water. The measured BET surface area and micropore volume are $1155 \mathrm{~m}^{2} \mathrm{~g}^{-1}$ and $0.23 \mathrm{~cm}^{3} \mathrm{~g}^{-1}$, respectively [4]. The coatings differed from 2.7 to $5.1 \mathrm{mg} \mathrm{cm}^{-2}$ carbon black. The holes were filled with sulfuric acid of various concentrations as electrolyte.

\subsubsection{Electrochemical Measurements}

All three parts of the cell were tightly mounted. Oxygen was supplied to the cathode at a flow rate of $22 \mathrm{~mL} \mathrm{~min}^{-1}$ at ambient pressure and the anode tank was filled with $4 \mathrm{M}$ aqueous methanol solution.

Current-voltage characteristics were measured simply by connecting anode and cathode with a voltmeter in parallel and an ampere meter in series with a variable resistance.

All supercapacitor measurements were performed with a potentiostat/galvanostat 7050 from AMEL Instruments. The SC was charged by three different methods: through a constant resistance, at constant current (galvanostatic) and at constant voltage sweep rate (cyclic voltammetry). For charging the fuel cell anode, for discharging the fuel cell cathode was electrically connected to the SC.

\subsection{Results for the Current-Voltage Characteristics}

The performance of the DMFC with and without the SC in between was tested (Figure 2). Operating the cell without the SC (both GDEs were fixed on the same Nafion ${ }^{\circledR} 212$ membrane) gave the best performance with $38(5) \mathrm{mW} \mathrm{cm}^{-2}$ maximum. This value is quite comparable to literature data for passive DMFCs, but for a semi-passive DMFC it seems 
to be worse [10, 14-17]. A reason for this might be that the GDE was not hot-pressed with the membrane and therefore the contact between them is not at its optimum. Another reason is that for each measurement under new conditions in the SC the cell needed to be opened. This led to mechanical stress on the catalyst layer and decreased its performance.

Adding the SC between the two fuel cell electrodes decreased the performance of the DMFC rapidly as a consequence of the higher internal resistance. $10 \mathrm{M}$ sulfuric acid as electrolyte gave the best performance (4.3(1) $\mathrm{mW}$ $\mathrm{cm}^{-2}$ ) and was therefore used as electrolyte for all measurements.

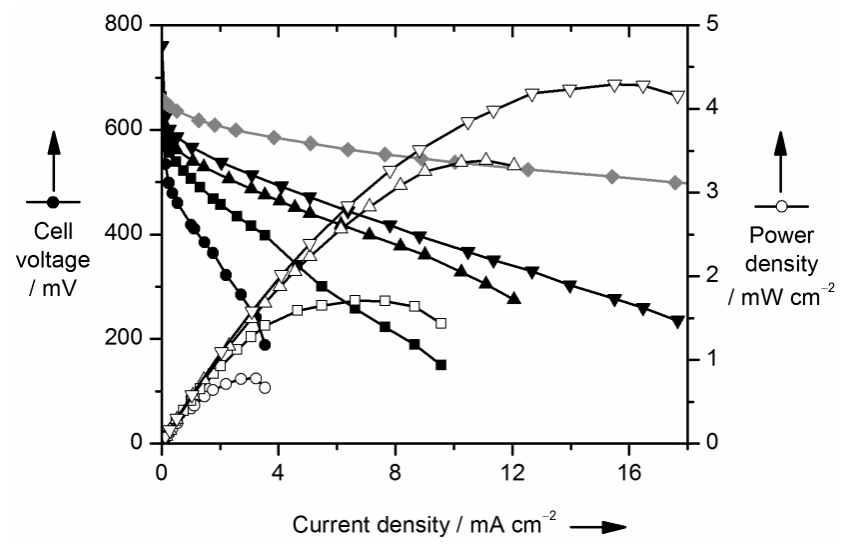

Figure 2: Voltage polarization and power curves of the semi-passive DMFC with different electrolytes in the inserted supercapacitor. Circles: $85 \% \mathrm{H}_{3} \mathrm{PO}_{4}$, squares: $1 \mathrm{M} \mathrm{H}_{2} \mathrm{SO}_{4}$, triangles up: $5 \mathrm{M} \mathrm{H}_{2} \mathrm{SO}_{4}$, triangles down: $10 \mathrm{M} \mathrm{H}_{2} \mathrm{SO}_{4}$, grey: DMFC in the absence of inserted supercapacitor material; the corresponding power performance curve is not shown.

\subsection{Resulting Supercapacitor Properties}

\subsubsection{Charging through a constant resistance}

Charging and discharging, shown in Figure 3, appears to be reversible. The first cycle is needed to bring the system into a defined state; therefore it is not included in the analysis. Before analyzing the results we have to discuss the thermodynamic driving force for the processes.

As mentioned in the concept, charging and discharging through a constant resistance takes place spontaneously without application of any external power. An open circuit voltage (OCV) of about $730 \mathrm{mV}$ was measured between anode and discharged SC. During the charging process this potential difference decayed exponentially to zero. It is thus the driving force for charging, and after charging the system is in thermodynamic equilibrium. The OCV between the fully charged SC and the cathode is about $650 \mathrm{mV}$. After discharging it is again zero. Forming or decomposing the electrical double layer at the SC interface changes its potential. The potential of the fuel cell electrodes is the same at the beginning and the end of charging and discharging process. Only the potential of the SC changes as a function of charging state. With this we define the relative potential of the SC between charged and uncharged state as the potential difference of the SC against the fuel cell electrodes. Now, we calculate the capacitance of the SC based on $C=Q / U$ where $Q$ is the accumulated charge and $U$ is the OCV. The charge is calculated by integrating the current vs. time curve.

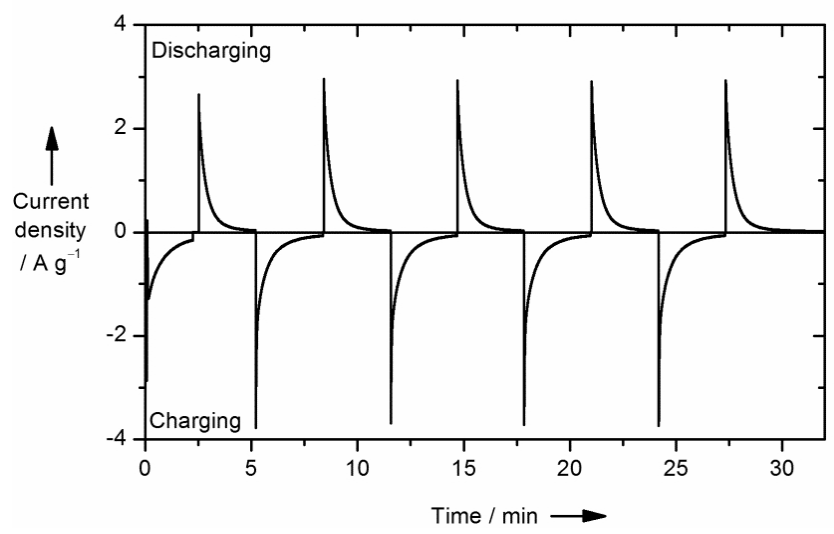

Figure 3: Charging/discharging cycles through a constant, external resistance (here $10 \Omega$ ).

With increasing external resistance $R_{\mathrm{SC}}$ the charge and, therefore, also the capacitance increase (Table 1). On charging through a low resistance the process might be diffusion or reaction limited by the anode (diffusion or oxidation of methanol) causing a diffusion or reaction overpotential which limits the total (integrated) charge. Increasing the external resistance decreases this overpotential. At the highest resistance the supercapacitor can reach its full capacity. 
Figure 4 shows that the total charge asymptotically reaches a maximum value which must be related to the maximum capacity of the supercapacitor. The phenomenological fit in Figure 4 (dashed line) shows that at the highest value of $R_{\mathrm{SC}}$ used in this experiment the total charge, and hence, the calculated capacitance, have nearly reached the maximum value.

There is a significant mismatch between the integrated charge from the charging and the discharging process. Such a loss has been observed previously, and it was shown that recombination of protons and electrons in the EDL on open circuit cannot account for these losses [4]. Its origin is still not understood.

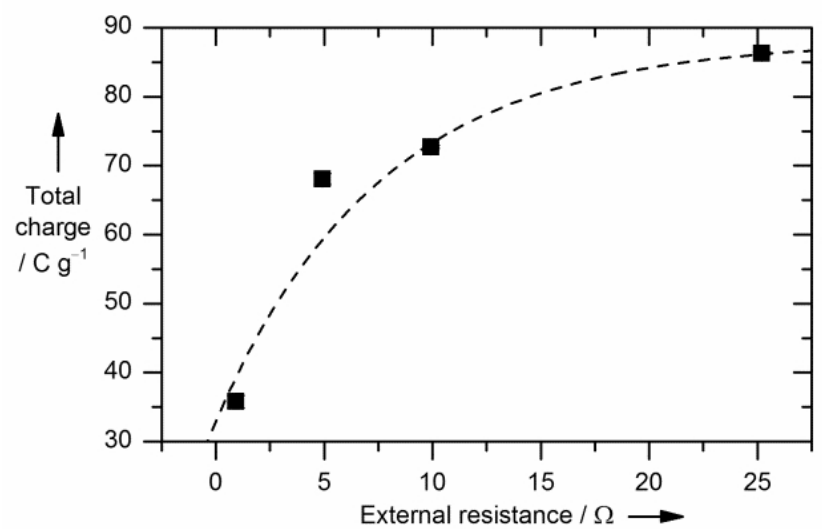

Figure 4: Total charge after charging through a constant, external resistance $R_{\mathrm{SC}}$. The dashed line is a phenomenological fit of exponentially decaying growth reaching a constant value of $88(4) \mathrm{C}^{-1}$.

Table 1: Integrated electric charge $Q$ in Coulomb (C) and capacitances $C$ in Farads (F) for different resistor values

\begin{tabular}{llllll}
\hline$R_{\mathrm{SC}}$ & \multicolumn{2}{c}{ Charging process } & \multicolumn{2}{c}{ Discharging process } & \multicolumn{2}{c}{ Losses } \\
$\Omega$ & $Q / \mathrm{C} \mathrm{g}^{-1}$ & $C / \mathrm{Fg}^{-1}$ & $Q / \mathrm{C} \mathrm{g}^{-1}$ & $C / \mathrm{F} \mathrm{g}^{-1}$ & $\mathrm{C} \mathrm{g}^{-1}(\%)$ \\
\hline 0.93 & $35.8(9)$ & $51(3)$ & $21.3(4)$ & $33(2)$ & $14.5(41 \%)$ \\
4.91 & $68.1(8)$ & $97(5)$ & $55.4(1)$ & $85(4)$ & $12.7(19 \%)$ \\
9.94 & $72.7(2)$ & $104(5)$ & $61.9(3)$ & $95(5)$ & $10.8(15 \%)$ \\
25.2 & $86.3(2)$ & $123(6)$ & $77.1(2)$ & $119(6)$ & $9.2(11 \%)$ \\
\hline
\end{tabular}

\subsubsection{Charging at constant current}

With charging the SC at constant current the resistance and voltage change with time, whereupon a linear behavior of the potential vs. time is expected. For our system, we observed two linear regimes (Figure 5). The duration of the first regime increases with decreasing current.

Experiments by Zerpa et al. using hydrogen instead of methanol at the anode show only a single linear regime [4]. Several processes take place and contribute to the voltage drop across the cell on charging the supercapacitor. They can be described by Ohmic resistances (contacts, electrolyte), $R C$ elements representing the porous anode as well as the capacitance of the supercapacitor, and diffusion or reaction overpotentials at the anode. Whereas the Ohmic resistance is time independent (no contribution to the slope) and the effect of the $R C$ element associated with the anode capacitance is expected to be negligible, the last two mentioned origins are expected to contribute to the slope. Diffusion normally leads to a pronounced transient regime followed by a steady state or a runaway of the voltage [18]. In the transient regime the ions are depleted at one boundary while ions build up at the other boundary. Therefore a driving force for migration arises until finally a steady state is reached. The duration of the transient period is a function of the current and so is the value of the diffusion overpotential. As the elements of the model are all in series the potentials simply sum up to yield the overall voltage.

The main deviations from ideal capacitative behavior take place at early times. This could be due to a more superficial capacitance associated with smaller time constants. Another explanation would be initial changes in overvoltage leading to drastically elevated slopes at higher currents and therefore to decreased apparent capacitances. As the processes causing these overvoltages approach steady state, their influence on the slope decreases. When hydrogen is used instead of methanol no mass transport (fast gas diffusion) or reaction (fast hydrogen oxidation) related process influences the slope; hence, only one linear regime from the capacitive behavior is observed. However, the current density was a factor 3 higher in the hydrogen experiments than for the $20 \mathrm{~mA}$ curve in Figure 5 so that the first regime could escape detection.

Above $500 \mathrm{mV}$, decomposition of water starts, and therefore the curve flattens further (Figure 5, $20 \mathrm{~mA}$ ). The capacitance is calculated from $C=I / m$ where $I$ is the current density $\left(\mathrm{A} \mathrm{g}^{-1}\right)$ and $\mathrm{m}$ the slope $\left(\mathrm{V} \mathrm{g}^{-1}\right)$. The results are collated in Section 2.4.4. 
An alternative explanation of the two slopes will be derived based on the cyclic voltammetry experiments in Section 2.4.3 and compared in Section 2.4.4.

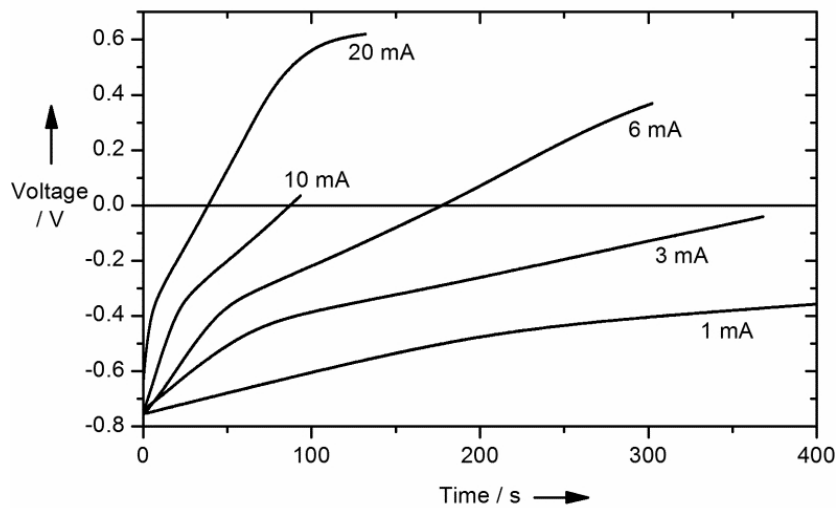

Figure 5: Galvanostatic charging curves at various constant currents.

\subsubsection{Charging at constant voltage sweep rate}

In cyclic voltammetry $(\mathrm{CV})$ the potential is controlled by a potentiostat. While the potential decreases exponentially in the fully spontaneous process, it decays linearly in CV. An exemplary cyclovoltammogram at $50 \mathrm{mV} \mathrm{s}^{-1}$ sweep rate is shown in Figure 6. The first cycle differs considerably from the following ones. For this first cycle the SC is fully discharged at the beginning. For the following cycles the SC stays partly charged and the system is in a more defined and stable state which leads to more reproducible cycles. The capacitance at different sweep rates is calculated from $C=I_{C} / v$, with $I_{C}$ being the capacitive current which equals the positive minimum of the $\mathrm{CV}$, and $v$ the sweep rate. The results are discussed in the next chapter.

Biesheuvel et. al. have investigated in detail the special effects occurring on capacitive charging of porous electrodes [19]. Measuring CV at different sweep rates provides different pieces of information. The formation of the double layer at the surface of a porous material can be separated into two processes which occur at different rates. The charging of the outer surface is quite fast as the ions do not have to migrate over a large distance. In contrast, charging the pores seems to take longer as the ions need to migrate inside, which might be inhibited by Coulomb repulsion. The sweep rate $v$ provides access to this time regime. At very fast sweep rates mainly the surface is charged as the ions do not have enough time to migrate into the pores. At very low sweep rates the ions can migrate into the pores and saturate the SC. Ardizzone et al. suggested convenient linear relations between the derived charge and the sweep rate for each of the two diffusion processes (equations (5) and (6)) [20]. An inverse proportional dependence of the sweep rate to the diffusion time is assumed. With a semi-infinite linear diffusion this leads to equation (5). As $Q$ is proportional to $v^{-1 / 2} Q^{-1}$ must be proportional to $v^{1 / 2}$ (equation (6)). Nevertheless, a further discussion of the equations is not reasonable as they are more phenomenological. More details can be found in reference [20]. The constants $k$ and $k$ ' are of no experimental interest. With $v$ extrapolating to infinite sweep rates near infinite $k / v^{1 / 2}$ becomes zero and $Q_{\text {surface }}$ can be calculated from the ordinate intercept (equation (5), Figure 7, left). In the same way, $Q_{\text {tot }}$ is obtained from the ordinate intercept of equation (6) by extrapolating $v$ to zero (Figure 7, right). The results are discussed in the following chapter.

$$
\begin{aligned}
& Q=Q_{\text {surface }}+\frac{k}{\sqrt{v}} \\
& \frac{1}{Q}=\frac{1}{Q_{\text {tot }}}+k^{\prime} \sqrt{v}
\end{aligned}
$$




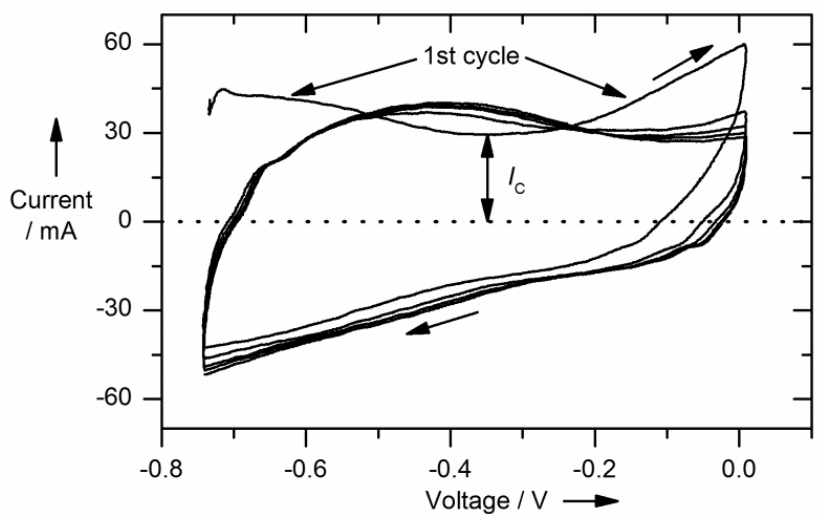

Figure 6: Exemplary cyclic voltammogram at $50 \mathrm{mV} \mathrm{s}^{-1}$ sweep rate.

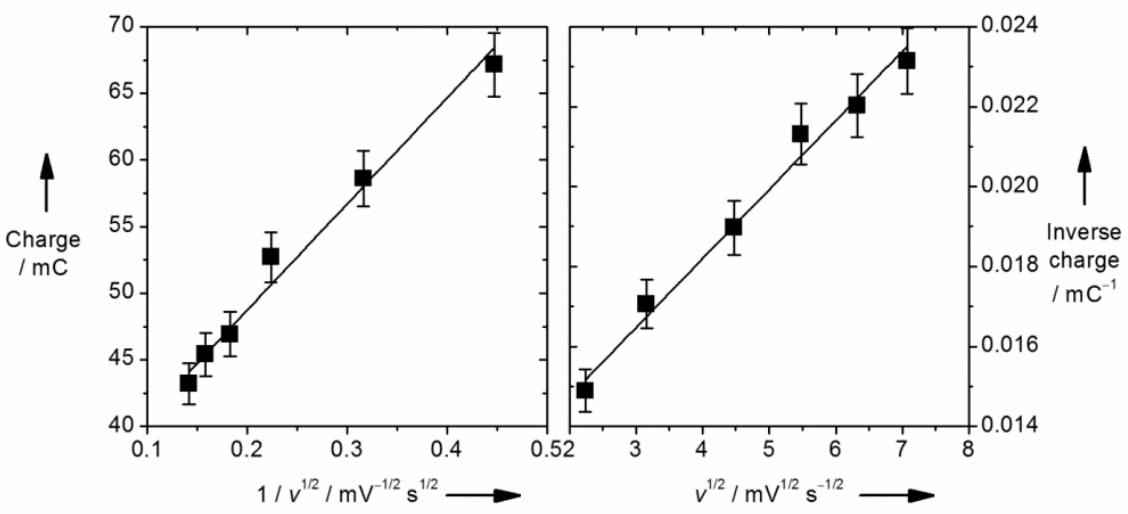

Figure 7: Plots of charge vs. sweep rate according to Ardizzone et al. [20].

\subsubsection{Comparison of the methods}

The results of the three methods at constant resistance $R_{\mathrm{SC}}$, constant voltage sweep rate (cyclic voltammetry) and constant current are collated in Table 2. The charges derived from the measurements at constant current and the surface and total charge obtained from Ardizzone's plots based on cyclic voltammetry are in good agreement. The surface charge is close to the same as the value obtained for the smallest constant resistance. The total charge matches very well the charge obtained at the highest resistance, i.e. the asymptotic value of $88(4) \mathrm{C} \mathrm{g}^{-1}$ from the exponential fit in Figure 4. We therefore conclude that for the spontaneous process at small resistances and therefore short charging times only the external surface is charged. The more the charging time is increased by increasing the resistance the more charge has time to diffuse inside the pores until the maximum value accessible by spontaneous charging is reached.

The above interpretation based on Ardizzone's plots leads to an alternative explanation of the two slopes if the constant current experiments. The processes of charging up the external surface and the internal surface of the pores are impressively and most directly seen as two regimes in the constant current experiments shown in Figure 5. The regime of the first linear range with higher slope up to the knee represents charging the external surface at early times, while the second regime represents the slower process of charging up the internal surface. In agreement with this interpretation, the capacitances calculated from the slopes of the first linear regime of the constant current measurements in Figure 5 and from the cyclic voltammograms are well compatible, except the value at $20 \mathrm{~mA}$ constant current. This is so because the cyclovoltammetric sweep rates are of the same order of magnitude as the charging rates at constant current. Hence, the two experiments result in the same (too low) capacitances at high constant currents as for high sweep rates.

In agreement with this, the capacitances obtained from the second linear regime with lower slope are the same at low currents as the values for slow spontaneous charging at the highest resistance where the supercapacitor approaches its full capacity.

Based on the BET surface area of $1155 \mathrm{~m}^{2} \mathrm{~g}^{-1}$ the value for the capacitance of $158 \mathrm{~F} \mathrm{~g}^{-1}$ derived at $1 \mathrm{~mA}$ from the slope of the second regime translates into $13.7 \mu \mathrm{F} \mathrm{cm}^{-2}$, which is close to the typical double layer capacitance of carbon blacks in aqueous electrolytes [21]. This confirms that at the end of the second regime the complete micropore surface contributes to the capacitance.

It seems clear that the two slopes in Figure 5 represent two different time-dependent processes. Which of the two above explanations is correct, i.e. whether the origin is related to diffusion and reaction kinetics at the anode or to free versus restricted diffusion outside and inside the supercapacitor electrode pores, has to remain open at this point. 
Table 2: Comparison of charges and capacitances derived from the three different techniques

\begin{tabular}{|c|c|c|c|c|c|c|c|}
\hline \multicolumn{3}{|c|}{ constant resistance } & \multicolumn{2}{|c|}{ cyclic voltammetry } & \multicolumn{3}{|c|}{ constant current } \\
\hline$R_{\mathrm{SC}} / \Omega$ & $Q / \mathrm{C} \mathrm{g}^{-1}$ & $C / \mathrm{F} \mathrm{g}^{-1}$ & $v / \mathrm{mV} \mathrm{s}^{-1}$ & $C / \mathrm{F} \mathrm{g}^{-1}$ & $I / \mathrm{mA}$ & $\begin{array}{l}1^{\text {st }} \text { slope } \\
C / \mathrm{F} \mathrm{g}^{-1}\end{array}$ & $\begin{array}{l}2^{\text {nd }} \text { slope } \\
C / \mathrm{F} \mathrm{g}^{-1}\end{array}$ \\
\hline 0.93 & $35.8(9)$ & $51(3)$ & 5 & $49(2)$ & 1 & $48(2)$ & $158(6)$ \\
\hline 4.91 & $68.1(8)$ & $97(5)$ & 10 & $50(2)$ & 3 & $41(1)$ & $155(6)$ \\
\hline 9.94 & $72.7(2)$ & $104(5)$ & 20 & $38(2)$ & 6 & $47(2)$ & $134(5)$ \\
\hline \multirow[t]{4}{*}{25.2} & $86.3(2)$ & $123(6)$ & 30 & $41(2)$ & 10 & $36(1)$ & $127(5)$ \\
\hline & & & 40 & $41(2)$ & 20 & $17(1)$ & $122(4)$ \\
\hline & & & 50 & $40(2)$ & & & \\
\hline & & & average & $43(5)$ & average & $43(5)^{*}$ & \\
\hline fit & & & $Q_{\text {surface }}=$ & $32(1) \mathrm{Cg}^{-1}$ & & & \\
\hline (Figure 4) & $88(4)$ & & $Q_{\mathrm{tot}}=$ & $89(3) \mathrm{Cg}^{-1}$ & & & \\
\hline
\end{tabular}

* italic value not used for calculating the average.

\subsection{Conclusions for Electrochemical Hydrogen Storage}

The method works reversibly at room temperature. The storage capacity, although an order of magnitude larger than for hydrogen physisorption on the same carbon material at the same temperature and pressure, is too small for technical applications. More importantly, proton diffusion presents a severe kinetic limitation, which leads to the requirement of very thin porous carbon layers.

A promising alternative hybrid setup without diffusion restrictions involves an external EDL supercapacitor which is charged up when the fuel cell produces extra energy and is discharged during peak power requirements.

\section{Hydrogen Production via On-board Electro-Reforming}

\subsection{The Concept}

Liquid fuel is far easier to store and transport than a gas, but on-board steam reforming is too complex for routine operation of a fuel cell in a car. An easy alternative consists in electrochemical conversion of methanol to $\mathrm{H}_{2}$ and $\mathrm{CO}_{2}$, which is close to isoenergetic [6]:

$$
\mathrm{CH}_{3} \mathrm{OH}_{\mathrm{aq}}+\mathrm{H}_{2} \mathrm{O} \rightarrow \mathrm{CO}_{2, \mathrm{~g}}+3 \mathrm{H}_{2}, \mathrm{~g} \quad \Delta G^{\circ}=-15 \mathrm{~kJ} \mathrm{~mol}^{-1} \quad E^{\circ}=+0.016 \mathrm{~V}
$$

Reaction (7) should occur spontaneously, but it is highly activated. A suitable catalyst can reduce the activation energy, and if conducted in a fuel cell type setup (with an inert gas replacing $\mathrm{O}_{2}$ on the cathode side) the reaction can be promoted further by application of an external voltage. The two gaseous products evolve separately at the anode and cathode side of the cell so that no separation is necessary.

The concept outlined in Figure 8 is that the fuel is carried along aboard the vehicle as a liquid, and that a fraction of the electrical power provided by the hydrogen fuel cell is used to overcome the activation energy of the electrode reaction for the conversion to $\mathrm{H}_{2}$. In this way, $\mathrm{H}_{2}$ can be provided on demand, perhaps in combination with intermediate gas phase storage to provide more flexibly to peak power demands.

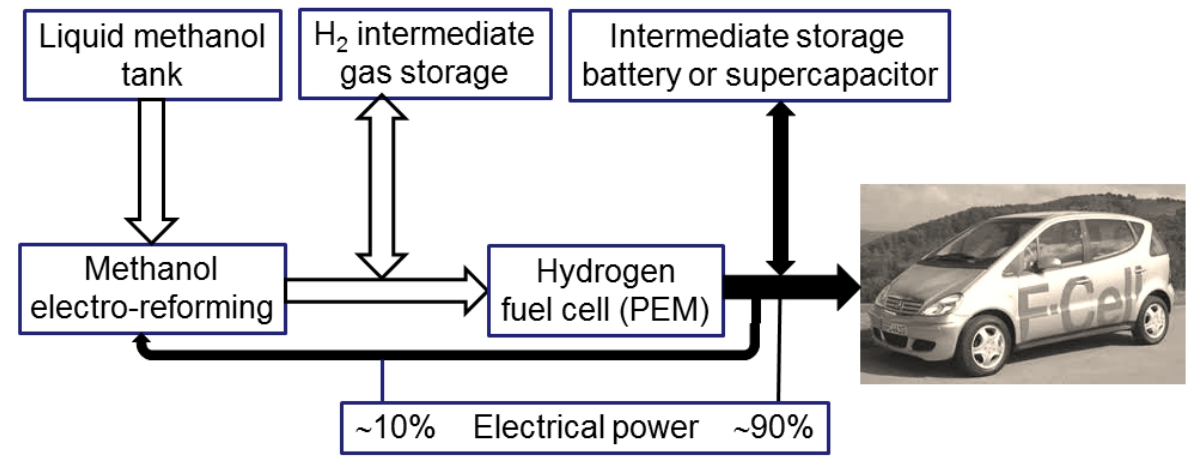

Figure 8: Scheme of on-board electrochemical methanol reforming that produces hydrogen for a PEM fuel cell. The aim is that a fraction of only $\sim 10 \%$ of the fuel cell power is needed to activate the electro-reforming. 


\subsection{Experimental details}

A commercial demonstrator DMFC from h-tec, unfortunately with undisclosed membrane and electrode composition, was used to investigate the electrochemical reforming. According to the manufacturer power densities of up to $3 \mathrm{~mW} \mathrm{~cm}^{-2}$ can be reached when the demonstrator is run in the intended DMFC mode. All experiments were performed in galvanostatic mode using an Amel 7050 bipotentiostat, and the voltages were measured using a Fluke 189 True RMS Multimeter. Both devices were interfaced to a PC using Win XP.

A Plexiglas ${ }^{\circledR}$ cap was machined and mounted on the cathode side to collect the evolving gas and measure it by means of an inverted burette that was initially filled with water and connected to a compensating pressure reservoir. The cathode compartment was prefilled with hydrogen gas at ambient pressure.

0.5 M, 1.0 $\mathrm{M}$ and 2.0 M aqueous methanol solutions were prepared using LiChrosolv methanol in bidistilled water. Faradaic efficiencies were calculated by relating the amount of evolved hydrogen to the time-integrated current. This fuel was recirculated through the cell by means of an electrical pump and regularly replaced to avoid any depletion of concentration.

\subsection{Results and Discussion}

The maximum efficiency of a direct methanol fuel cell operated with $2 \mathrm{M}$ aqueous methanol is temperature dependent and amounts to $19 \%$ at a temperature of $313 \mathrm{~K}$ and a current density of $150 \mathrm{~mA} \mathrm{~cm}^{-2}$ [22]. This efficiency is to be compared with that of a PEM fuel cell operated with hydrogen from an autothermal methanol reformer. Geissler $e t$ al. found that only $4 \%$ of the methanol's energy content is lost during the reforming and gas clean-up process (preferential CO oxidation) [23]. Only about 50\% of the hydrogen energy is converted to electrical energy, and about $20 \%$ of the generated electric power is used for driving auxiliary equipment (compressors, pumps). This reduces the net electrical energy that is available for the vehicle to $33 \%$ of the energy content in the methanol feed. Both the DMFC and in particular the autothermal reformer combined with hydrogen PEM thus compare favorably with the $17 \%$ fuel cycle efficiency of current state-of-art internal combustion engines [24].

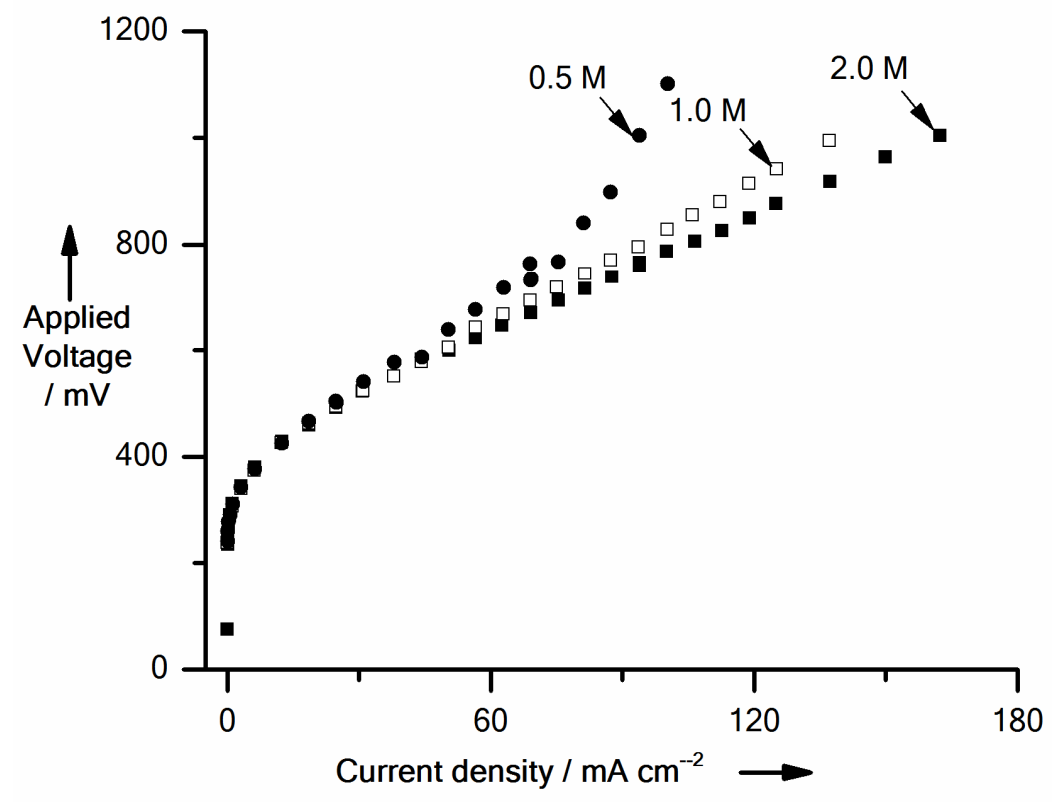

Figure 9: Voltage-current curves of methanol electro-reforming at $293 \mathrm{~K}$.

Figure 9 plots the applied voltage against current of our experiments at $293 \mathrm{~K}$. For comparison with the above efficiencies of DMFC and hydrogen PEM with thermal methanol reformer we assume electro-reforming of methanol at $492 \mathrm{mV}$, giving a current density of $25 \mathrm{~mA} \mathrm{~cm}^{-2}$. We further assume that the PEM is operated at $750 \mathrm{mV}$, corresponding to an efficiency of $50.6 \%$ and a current density of $250 \mathrm{~mA} \mathrm{~cm}{ }^{-2}$ [24]. Based on these voltages, the overall efficiency is then $20.6 \%$ since slightly more than $1 / 3$ of the fuel cell power is available for the vehicle. In order to realize steady-state operation with equal current in both devices the reformer based on the demonstrator needs an active area 10 times as large as the hydrogen fuel cell.

The low production rate can be explained with the inferior current capability of the demonstrator. The device is rated with a power capability of only $3 \mathrm{~mW} \mathrm{~cm}^{-2}$ when used in DMFC mode. The reformer built from the discussed DMFC could be shown to be able to produce nearly the same power when combined with a ten times smaller hydrogen fuel cell. The low current density of the demonstrator and the derived reformer could be explained with a small BET 
surface and low catalyst loadings on the anode. Anyway, the discussed setup with the improvised electrochemical reformer was almost able to reach the power densities promised for DMFC mode. Good efficiencies were also obtained for a constant load. If the applied voltage could be reduced even more this would change the picture dramatically, considering that electro-reforming is far less complex than steam reforming. Recently, promising novel, efficient homogeneous ruthenium complex catalysts have been developed for aqueous phase methanol dehydrogenation to hydrogen and carbon dioxide $[25,26]$. It remains to be seen whether they can also be used as electrocatalysts in the above electro-reforming process, marking the next step towards a methanol economy.

\section{Conclusions}

We have analyzed two options for the use of methanol in electrochemical energy conversion and storage. In a modified DMFC with integrated large surface area supercapacitor protons and electrons can be stored reversibly in an electrical double layer. This can be viewed as an alternative way of hydrogen storage which provides a buffer to adapt flexibly to cyclic energy demand. Because it involves diffusion of protons the method is limited to thin layers of supercapacitor materials. A set-up in which a conventional supercapacitor is wired up in parallel rather than in series with the DMFC electrodes is preferred since it would respond faster and avoid extra resistance in the DMFC.

Secondly, we have analyzed electro-reforming of methanol for providing hydrogen flexibly. The concept is attractive because of its simplicity compared with thermal steam reforming. However, with conventional anode electrocatalysts the activation of the reaction is still high and leads to severe losses for the process which according to thermodynamic criteria is spontaneous. Nevertheless, it was shown that the current densities reached are comparable to those of an equivalent DMFC with the same catalyst loading and membrane electrode assembly. This can easily be understood as it is know that hydrogen reduction and oxidation are very facile in comparison to methanol oxidation [2729]. Therefore the additional step comes with virtually no efficiency penalty. Furthermore, in a typical application scenario, the reformer makes decoupling of the sluggish methanol from load peaks possible. As hydrogen fuel cells provide superior efficiencies at high power densities and the reformer could stay in its sweet spot, the overall setup would attain higher efficiencies than a comparable DMFC and necessitate less catalyst and therefore less precious metal.

\section{References}

1 U. Eberle, M. Felderhoff, F. Schüth, Angew. Chem. Int. Ed., 2009, 48, 6608.

2 W. I. F. David, Faraday Discuss. 2011, 151, 399.

3 L. Dienberg, R. Haug, G. Rauhut, E. Roduner, Phys. Chem. Chem. Phys. 2013, 15, 5836.

4 J. E. Zerpa Unda, E., Roduner, 2012, Phys. Chem. Chem. Phys., vol. 14, pp. 3816-3824.

5 Department of Energy - United States of America, On-board fuel processing go/no-go decision: DOE Decision Team Committee Report, 2004. http://wwwl.eere.energy.gov/hydrogenandfuelcells/pdfs/committee_report.pdf.

6 C. R. Cloutier, D.P. Wilkinson, Int. J. Hydrogen Energy, 2010, 35, 3967.

7 K. Kinoshita, in Carbon: Electrochemical and physicochemical properties, John Wiley \& Sons, New York, 1988, 93.

8 V. V. Panic, R. M. Stevanovic, V. M. Jovanovic, A. B. Dekanski, J. Power Sources 2008, 181, 186.

9 K.-A. Starz, R. Zuber, A. Krämer, K. Fehl, J. Köhler, S. Wittpahl, in European Patent Specification, Vol. EP 1176 652 B1, Germany, 2002.

10 J. G. Liu, T. S. Zhao, R. Chen, C. W. Wong, Electrochem. Commun., 2005, 7, 288.

11 J. G. Liu, T. S. Zhao, Z. X. Liang, R. Chen, J. Power Sources, 2006, 153, 61.

12 K.-W. Park, H.-J. Ahn, Y.-E. Sung, J. Power Sources, 2002, 109, 500.

13 T. Yuan, Z. Q. Zou, M. Chen, Z. L. Li, B. J. Xia, H. Yang, J. Power Sources, 2009, 192, 423.

14 D. J. Kim, E. A. Cho, S. A. Hong, I. H. Oh, H. Y. Ha, J. Power Sources, 2004, 130, 172.

15 Q. Z. Lai, G. P. Yin, Z. B. Wang, Int. J. Energy Research, 2009, 33, 719.

16 J. G. Liu, G. Q. Sun, F. L. Zhao, G. X. Wang, G. Zhao, L. K. Chen, B. L. Yi, Q. Xin, J. Power Sources, 2004, 133, 175.

1717 X. G. Wang, J. H. Liao, C. P. Liu, W. Xing, T. H. Lu, Electrochem. Commun., 2009, 11, 19

18 P. Delahay, G. Mamantov, Anal. Chem. 1955, 27, 478.

19 R. Zhao, M. van Soestbergen, H.H.M. Rijnaarts, A. van der Wal, M.Z. Bazant, P.M. Biesheuvel, J. Colloid Interface Sci., 2012, 384, 38.

20 S. Ardizzone, G. Fregonara, S. Trasatti, Electrochim. Acta, 1990, 35, 263.

21 M. Boero, T. Ikeshoji and K. Terakura, ChemPhysChem, 2005, 6, 1775.

22 S. H. Seo, C. S. Lee, Appl. Energ., 2010, 87, 2579.

23 K. Geissler, E. Newson, F. Vogel, T.-B. Truong, P. Hottinger, A. Wokaun, Phys. Chem. Chem. Phys., $2001,3,289$.

24 L. Gubler, H. Kuhn, T. J. Schmidt, G. G. Scherer, H.-P. Brack, K. Simbeck, Fuel Cells, 2004, 4, 196.

25 M. Nielsen, E. Alberico, W. Baumann, H.-J. Drexler, H. Junge, S. Gladiali, M. Beller, Nature, $2013,495,85$.

26 R. E. Rodriguez-Lugo, M. Trinicado, M. Vogt, F. Tewes, G. Santiso-Quinones, H. Grützmacher, Nature Chemistry, 2013, 5, 342 . 
27 C. R. Cloutier, D. P. Wilkinson, Int. J. Hydrogen Energ. 2010, 35, 3967.

28 F. Barbir, PEM fuel cells: Theory and practice, Elsevier Academic Press, Amsterdam; Heidelberg [u.a.] 2005.

29 J. Wu, X. Z. Yuan, H. Wang, M. Blanco, J. J. Martin, J. Zhang., Int. J. Hydrogen Energ. 2008, 33, 1735. 